\section{Deficiency of hyccin, a newly identified membrane protein, causes hypomyelination and congenital cataract}

Federico Zara ${ }^{1}$, Roberta Biancheri ${ }^{1}$, Claudio Bruno ${ }^{1}$, Laura Bordo $^{1}$, Stefania Assereto ${ }^{1}$, Elisabetta Gazzerro ${ }^{1}$, Federica Sotgia ${ }^{1,2}$, Xiao Bo Wang ${ }^{2}$, Stefania Gianotti ${ }^{1}$, Silvia Stringara ${ }^{1}$, Marina Pedemonte ${ }^{1}$, Graziella Uziel $^{3}$, Andrea Rossi ${ }^{4}$, Angelo Schenone ${ }^{5}$, Paolo Tortori-Donati ${ }^{4}$, Marjo S van der Knaap ${ }^{6}$, Michael P Lisanti ${ }^{1,2}$ \& Carlo Minetti ${ }^{1}$

We describe a new autosomal recessive white matter disorder ('hypomyelination and congenital cataract') characterized by hypomyelination of the central and peripheral nervous system, progressive neurological impairment and congenital cataract. We identified mutations in five affected families, resulting in a deficiency of hyccin, a newly identified 521-amino acid membrane protein. Our study highlights the essential role of hyccin in central and peripheral myelination.

White matter disorders (WMDs) constitute a heterogeneous group of genetic and acquired diseases predominantly affecting the white matter of the brain. In the last decade, the genetic etiology of several WMDs has been elucidated. Nevertheless, about half of the cases remain without a specific diagnosis ${ }^{1}$. The largest single category among the unclassified WMDs consists of individuals with hypomyelination ${ }^{2}$.

We identified ten individuals from five families characterized by congenital cataract, progressive neurological impairment and diffuse myelin deficiency as shown by brain magnetic resonance imaging (MRI). All individuals presented with bilateral cataract at birth or in the first 2 months of life and underwent ocular surgery. Initial psychomotor development was normal. At the end of the first year of life, development was delayed in all individuals. All achieved the ability to walk only with support. Over time, the individuals experienced slowly progressive pyramidal and cerebellar dysfunction, muscle weakness and wasting prevailingly in the lower limbs and most became wheelchair bound. Mental deficiency ranged from mild to moderate (Supplementary Table 1 online). Brain MRI showed abnormalities consistent with diffuse cerebral hypomyelination ${ }^{3}$, whereas the cortex and deep gray matter structures were preserved (Fig. 1a,b). Neurophysiological studies demonstrated peripheral neuropathy in nine of the ten individuals. Motor nerve conduction velocity was slightly to markedly slowed depending on the age of individuals, and the amplitude of compound muscle action potentials was also reduced. A sural nerve biopsy, performed in four individuals, showed deficiency of the myelin sheath in several nerve fibers and mild axonal loss, without active axonal degeneration (Fig. 1c,d). This

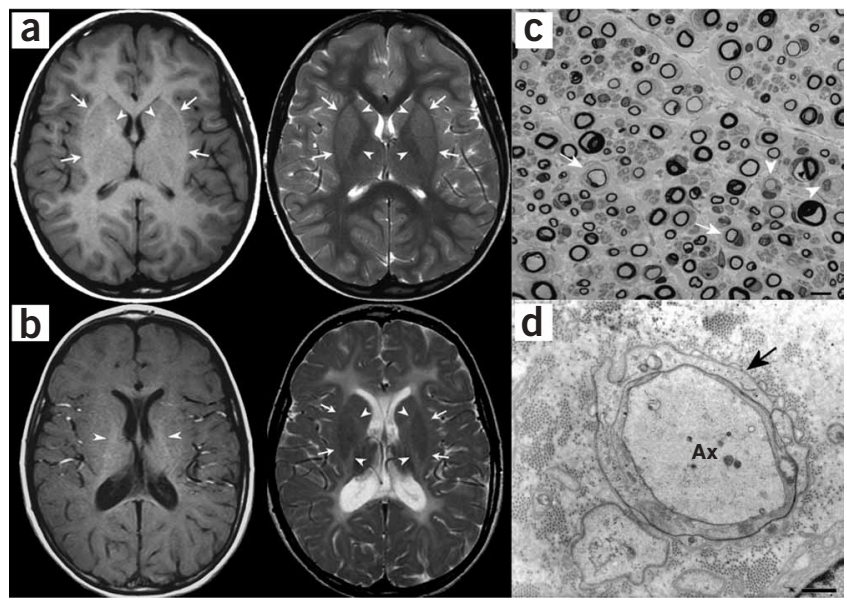

Figure 1 Brain MR images and sural nerve biopsy. (a) Magnetic resonance images of a healthy 8-year-old subject. The high signal of the internal (arrowheads) and external capsules (arrows), cerebral hemispheric white matter on the T1-weighted images (left), and the low signal intensity of these structures on the T2-weighted images (right) indicate a normal myelin content of the brain. (b) Magnetic resonance images of individual 6 at 8 years of age. On the T1-weighted images (left), a faint increase in signal intensity is seen only at the genu of the internal capsules (arrowheads), whereas the remaining white matter shows the same signal intensity as the cortex. On the T2-weighted images (right), the internal capsules (arrowheads), external capsules (arrows) and hemispheric white matter show higher signal intensity than in normal individuals. This signal behavior is indicative of hypomyelination. (c) Light microscopy of the sural nerve biopsy of individual 2. Semithin sections stained with toluidine blue showing a decreased number of myelinated fibers and the presence of several large axons lacking a myelin sheath (arrowheads) or surrounded by a thin myelin sheath; occasionally, small onion bulbs formed by Schwann cell processes wrapping around thinly myelinated axons are observed (arrows), indicative of remyelination. Bar: $5 \mu \mathrm{m}$. (b) Electron microscopy of the sural nerve biopsy of individual 2. Thin sections, stained with lead citrate and uranyl acetate show some axons surrounded by a few uncompacted myelin lamellae. Arrow points to defective axonal $(\mathrm{Ax})$ myelination. Bar: $1 \mu \mathrm{m}$. This study was approved by the G. Gaslini Ethics Committee. Written, informed consent was obtained from all adult participants and from parents on behalf of their children.

${ }^{1}$ Muscular and Neurodegenerative Disease Unit, G. Gaslini Institute and University of Genova, Italy. ${ }^{2}$ Department of Cancer Biology and Department of Biochemistry and Molecular Biology, Thomas Jefferson University, Kimmel Cancer Center, Philadelphia, Pennsylvania, USA. ${ }^{3}$ Division of Child Neuropsychiatry, C. Besta Institute, Milan, Italy. ${ }^{4}$ Department of Pediatric Neuroradiology, G. Gaslini Institute, Genova, Italy. ${ }^{5}$ Department of Neurosciences, Ophthalmology and Genetics, University of Genova, Genova, Italy. ${ }^{6}$ Department of Child Neurology, VU University Medical Center, Amsterdam, The Netherlands. Correspondence should be addressed to F.Z. (federicozara@ospedale-gaslini.ge.it) and C.M. (minettic@unige.it). 
a

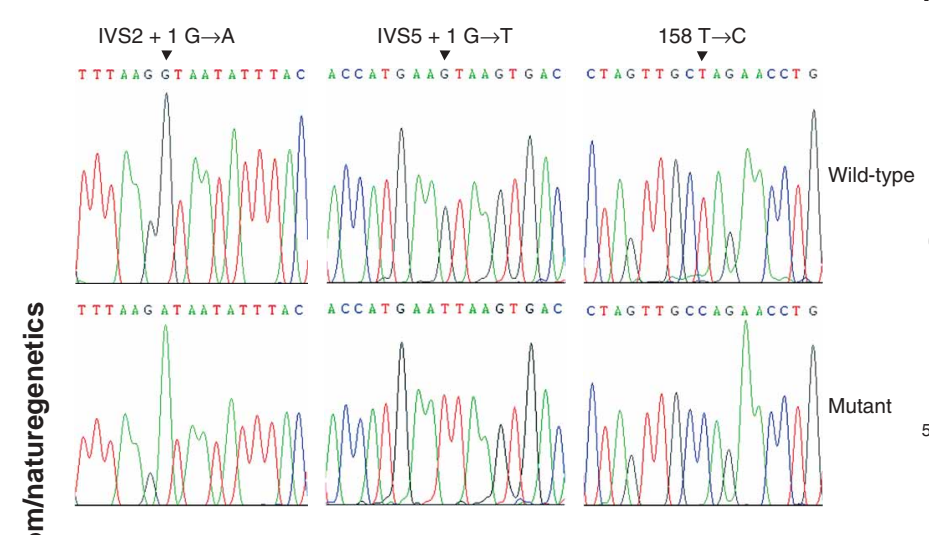

b

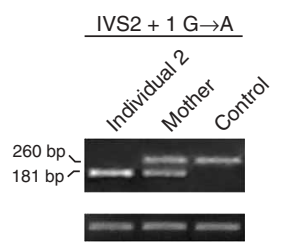

C

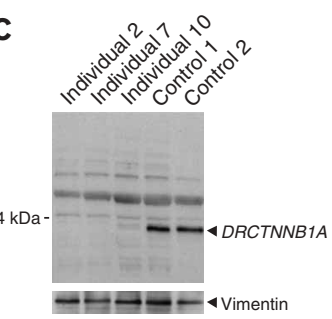

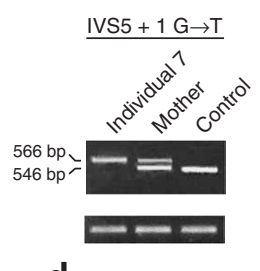

d

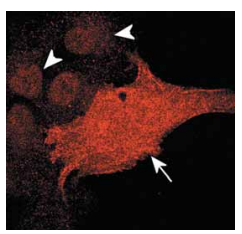

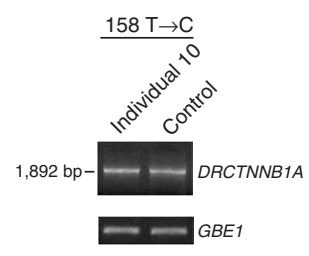

e

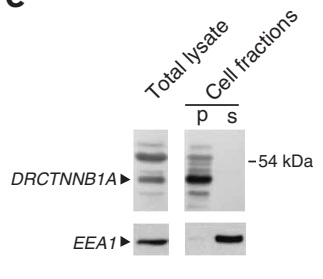

Figure 2 Characterization of mutations and subcellular localization of DRCTNNB1A. (a) Electropherograms showing wild-type and mutant alleles. (b) RT-PCR analysis of DRCTNNB1A showing aberrant mRNA for Mut1 and Mut2. GBE1 (glycogen branching enzyme) was amplified as internal control. (c) Immunoblot analysis of hyccin. Lanes 1-3: total cellular lysates of fibroblasts from affected individuals. Lane 4-5: controls. In individuals 2 and 7, hyccin is absent, whereas individual 10 shows a residual amount of the protein ( 5\%). An antibody against vimentin was used as an internal control. (d) Immunofluorescence analysis of myc-tagged hyccin in transfected COS-7 cells using a monoclonal antibody to myc showing a patchy localization of the protein at the plasma membrane of a transfected cell (arrow). Adjacent untransfected cells are indicated by arrowheads. (e) Immunoblotting of soluble and particulate fractions obtained from alkaline carbonate extraction ( $\mathrm{pH} \mathrm{11)} \mathrm{of} \mathrm{human} \mathrm{control} \mathrm{fibroblasts} \mathrm{using} \mathrm{polyclonal} \mathrm{antibody} \mathrm{to} \mathrm{hyccin.} \mathrm{Hyccin} \mathrm{localizes} \mathrm{in} \mathrm{the} \mathrm{particulate}$ fractions containing high-affinity membrane proteins. Early endosome antigen 1 (EEA1), which is peripherally associated with the membrane ${ }^{5}$, is rendered soluble by alkaline sodium carbonate and is used as an internal control. P indicates the particulate fraction and S the soluble fraction.

constellation of clinical, neuroradiological, neurophysiological and neuropathological findings was not consistent with any of the known WMDs. Therefore, we hypothesized that it represents a novel autosomal recessive WMD, which we have termed 'hypomyelination and congenital cataract' (HCC).

To identify the causative gene, we performed a genome-wide scan with $10 \mathrm{cM}$-spaced microsatellite markers in three consanguineous families (families 2, 3 and 5; Supplementary Fig. 1 and Supplementary Methods online). We identified a unique segment of homozygosity on chromosome 7p21.3-p15.3 between markers D7S517 and D7S510. Parametric linkage analysis showed a cumulative maximum lod score of 4.53 (Supplementary Table 2 online). We genotyped the remaining families and found homozygosity for this locus in all individuals. The typing of additional markers refined the critical homozygous region to $4.4 \mathrm{Mb}$ between markers D7S503 and D7S673 (Supplementary Fig. 1). The HCC locus contained 31 transcriptional units, mainly of unknown function. The systematic screening of all transcripts of the critical region led to the identification of two mutations affecting a splice site (IVS2+1G $\rightarrow$ A (Mut1) in families 1-3 and IVS5+1G $\rightarrow$ T (Mut2) in family 4) and a missense mutation (158T $\rightarrow \mathrm{C}$ (Mut3) in family 5) in DRCTNNB1A (Fig. 2a and Supplementary Methods).

Mutl causes skipping of exon 2, which harbors the initiation codon (Fig. 2b) and probably results in the lack of protein production. By introducing a cryptic splice site downstream in intron 5, Mut2 leads to the insertion of a 20-bp intronic segment into the DRCTNNB1A mRNA, resulting in a premature stop codon at position 145 (Fig. 2b and Supplementary Methods). Mut3 results in the substitution of a leucine residue with a proline at position 53 of the protein (L53P), without altering the structure of the DRCTNNB1A mRNA (Fig. 2b). We did not identify these mutations in 400 control chromosomes. Sequence alignment of the protein encoded by human DRCTNNB1A and its homologs in different species indicated that the leucine residue affected by Mut3 is highly conserved throughout evolution (Supple- mentary Fig. 2 online). Therefore, the L53P variant is most likely pathogenic.

In order to explore the effect of the identified mutations, we raised polyclonal rabbit antibodies against the $\mathrm{N}$ terminus of the protein encoded by human DRCTNNB1A. Immunoblot analysis showed that it is absent or strongly reduced in fibroblast lysates from individuals carrying the three different mutations (Fig. 2c and Supplementary Methods). In individual 10 (Mut3), who is still walking with support at age 20 years and does not have a peripheral neuropathy, a residual amount of the protein is present. These data indicate that HCC is caused by the deficiency of the protein encoded by $D R C T N N B 1 A$ and suggest a correlation between reduced protein level and phenotypic severity.

DRCTNNB1A encodes a 521-amino acid protein, which we have named hyccin. A search in the protein database showed the presence of putative hyccin orthologs in different species and a human paralog on chromosome 2q33.1 (Supplementary Fig. 2). RNA blot analysis showed that DRCTNNB1A is expressed in several adult tissues and is ubiquitously expressed in the brain (Supplementary Fig. 3 online). Using RT-PCR, we also found that DRCTNNB1A is expressed in the lens (Supplementary Fig. 3). InterProScan and PROSITE analysis of hyccin did not identify any known functional domains or motifs. TMpred analysis uncovered two putative transmembrane segments (Supplementary Fig. 4 online), suggesting a membrane localization. DRCTNNB1A has been shown to be downregulated by $\beta$-catenin in colon carcinoma (SW480) cells and its expression has been found to be decreased in different colorectal cancer tissues ${ }^{4}$. These findings suggest a potential role of $D R C T N N B 1 A$ in tumorigenesis. However, the function of DRCTNNB1A has remained unknown ${ }^{4}$.

We next subcloned the full-length wild-type DRCTNNB1A cDNA into a Myc-tagged expression vector and transfected the construct into COS-7 cells. Immunocytochemical staining of the transfected cells with monoclonal antibodies to Myc detected hyccin at the plasma membrane with a patchy distribution (Fig. 2d and Supplementary Fig. 5 and Supplementary Methods online). We further explored the 
membrane association of hyccin by cell fractionation ${ }^{5}$. In control fibroblasts, hyccin is present in the particulate fraction containing high-affinity membrane-binding proteins, whereas it is absent in the soluble fraction containing cytosolic or low-affinity membrane binding proteins (Fig. 2e and Supplementary Methods). These data provide evidence that hyccin is a tightly associated cell membrane protein.

In conclusion, we describe a new autosomal recessive disorder characterized by a central and peripheral myelin deficiency and congenital cataract. We show that this disorder is caused by the defect of a newly identified cell membrane protein encoded by DRCTNNB1A. Our data indicate that hyccin is essential for proper myelination in both the central and peripheral nervous system. The process of myelination is complex and occurs as an interplay between glial cells and neurons. Consequently, the pathogenesis of myelin disorders is highly heterogeneous. A molecular link between cerebral and peripheral myelination disorders and congenital cataract has been provided by gap junctions proteins ${ }^{6-9}$. The hyccin protein product provides the second molecular link. Recent data indicate that $\beta$-catenin-mediated Wnt signaling is involved in both gliogenesis $^{10,11}$ and lens epithelial differentiation ${ }^{12,13}$. Notably, it has been shown that $\beta$-catenin also suppresses the expression of myelin oligodendrocyte markers such as myelin basic protein and proteo을 lipid protein 1 (ref. 11). Further studies will be required to uncover the role of hyccin and the mechanisms underlying the abnormal myelination in HCC.

\section{ACKNOWLEDGMENTS}

We thank R. Gatti, P. Palazzolo, V. Costa and the families of the affected individuals for their collaborative efforts and P. Broda for technical assistance.

This work has been supported by the Pierfranco and Luisa Mariani Foundation ONLUS (grant R-04-37), the Telethon Italy Foundation (grant GGP05156), the Istituto Superiore della Sanità (grant 526/A32) and a donation from R. Bulgarelli. F.S. and M.P.L. were supported by grants from the US National Institutes of Health and the American Heart Association. The samples were obtained from the 'Cell Line and DNA Bank from Patients Affected by Genetic Diseases' collection (http://www.gaslini.org/labdppm.htm) supported by Italian Telethon grants (project number GTF04002).

\section{AUTHOR CONTRIBUTIONS}

F.Z.: study design, supervision of the molecular study, writing of the manuscript; L.B. and S.G.: linkage analysis, mutational screening, RNA analysis; R.B., C.B., G.U., M.S.v.K: phenotype assessment; S.A., E.G., S.S.: immunoblot and cell fractionation; F.S., X.B.W., M.P.L.: subcellular localization and immuofluorescence; A.S.: nerve biopsy pathology; A.R. and P.T.-D.: neuroradiological evaluation; C.M.: study design, supervision of the clinical study, writing of the manuscript.

\section{COMPETING INTERESTS STATEMENT}

The authors declare that they have no competing financial interests.

Published online at http://www.nature.com/naturegenetics

Reprints and permissions information is available online at http://npg.nature.com/ reprintsandpermissions/

1. Schiffmann, R. \& van der Knaap, M.S. Curr. Opin. Neurol. 17, 187-192 (2004).

2. van der Knaap, M.S. et al. Radiology 213, 121-133 (1999).

3. van der Knaap, M.S. et al. AJNR Am. J. Neuroradiol. 23, 1466-1474 (2002).

4. Kawasoe, T. et al. Cancer Res. 60, 3354-3358 (2000).

5. Mu, F.T. et al. J. Biol. Chem. 270, 13503-13511 (1995).

6. Uhlenberg, B. et al. Am. J. Hum. Genet. 75, 251-260 (2004)

7. Mackay, D. et al. Am. J. Hum. Genet. 64, 1357-1364 (1999).

8. Shiels, A. et al. Am. J. Hum. Genet. 62, 526-532 (1998).

9. Bergoffen, J. et al. Science 262, 2039-2042 (1993).

10. Shimizu, T. et al. Dev. Biol. 282, 397-410 (2005).

11. Kasai, M. et al. Genes Cells 10, 777-783 (2005).

12. Stump, R.J. et al. Dev. Biol. 259, 48-61 (2003).

13. Lyu, J. \& Joo, C.K. Development 131, 1813-1824 (2004). 